\title{
The Effect of Five Weeks of Basic Military Training on Physical Fitness and Blood Biochemical Factors in Obese Military Recruits Just Conscripted into the Army
}

\author{
Sang Gook Seo ${ }^{1}$ PhD, Se Hwan Park ${ }^{2}$ PhD, Jin-Hwan Yoon ${ }^{2}$ PhD, Byeong-Wan Kim ${ }^{3}$ PhD, Hyunseok Jee ${ }^{4}$ PhD \\ ${ }^{1}$ Graduate School of Business and National Defense Strategy, Hannam University, Daejeon; ${ }^{2}$ Department of Sports Science, Hannam University, Daejeon; ${ }^{3}$ Division of \\ Sports Science, Daejeon University, Daejeon; ${ }^{4}$ School of Kinesiology, Yeungnam University, Gyeongsan, Korea
}

PURPOSE: The purpose of this study was to investigate the effects of 5 weeks of basic military training on the physical fitness and blood biochemical factors in obese military recruits.

METHODS: Forty-eight male recruits (20-25 years of age) were randomly assigned to the Control group and Exercise group. Both groups completed 5 weeks of basic military training. The Exercise group additionally performed a resistance exercise twice daily in the morning and afternoon (1-3 sets, 3 days per week) and power walking (60-80\% HRmax, 30 minutes) four times weekly. Four body composition variables, physical fitness factors, serum lipid profiles, and obesity-related hormone levels were analyzed.

RESULTS: Body weight, body fat ratio, body mass index, and waist circumference were significantly decreased in both groups between pre- and post-exercise tests $(p<.01)$. However, no changes in these parameters were observed between the Control and Exercise groups. Twenty-meter shuttle running and maximal oxygen uptake were significantly increased post exercise $(p<.001)$. Total cholesterol, triglyceride, and low-density lipoprotein levels were significantly decreased in both (Control and Exercise) post-exercise tests $(p<.05)$. Leptin in both groups was significantly decreased; however, ghrelin showed a reverse pattern $(p<.01)$. A significantly changed maximal oxygen uptake value via training was noted in both groups $(p<.001)$. The time and interaction between the groups and the time were also significantly affected $(p<.001)$.

CONCLUSIONS: Our findings suggest that an additional exercise-training program with the basic military training plays an important role in improving military fitness and combat power.

Key words: Physical fitness, Military training, Body compositions, Isometric force, Obesity-related markers

\section{INTRODUCTION}

According to the 2014 annual report of the Korea National Health \& Nutrition Examination Survey, two out of five men and one out of four women are obese, and the obesity rate for men in their 20 s is $29.3 \%$, which is higher than that for women (14.4\%). Sports-related scientists define obesity as a kind of metabolic syndrome that is associated with complications, such as type 2 diabetes, hyperlipidemia, coronary heartrelated diseases, and cancers, and is involved in the physical function and fitness decline $[1,2]$. The obesity rate increase in our societies can be raised as not only a socioecological public health related issue, but also as an unexceptionally military issue (e.g., possibly discharged from military service from obesity-derived health problems, physical fitness-related health level of each soldier is directly related to the national defense force), which can be serious since the army exists for the security of the whole country and its citizens [3,4]. We thus emphasized researching the current physical fitness of military recruits and how to improve their physical fitness (if insufficient) with a decreasing fat rate to strengthen

Corresponding author: Hyunseok Jee Tel +82-53-810-3139 Fax 82-53-810-3139 E-mail jeehs@ynu.ac.kr

Received 10 Mar 2020 Revised 4 Apr 2020 Accepted 23 May 2020

@) This is an Open Access article distributed under the terms of the Creative Commons Attribution Non-Commercial License (https://creativecommons.org/licenses/by-nc/4.0/) which permits unrestricted non-commercial use distribution, and reproduction in any medium, provided the original work is properly cited. 
our national defense force, which is initiated from each recruited military soldier (This is the rationale for selecting military recruits as the participants in this study). The personnel pool of military recruits originated from high-school graduates; their current various physical fitnessrelated index levels (body composition, muscular, and cardiovascular endurances, sprint ability, coordination, etc.) were evaluated [5]. Obesity rates have increased by $9.4 \%$ in 2002 and by $11.2 \%$ in 2008; furthermore, the physical fitness level was lowered by $8 \%$ in the first level vs. $11 \%$ higher in the 5th level, showing an increasing rate of related pathological symptoms such as hyperlipidemia, hypertension, and diabetes in the newly recruited military population [6]. However, the physical fitness improvement programs for military recruits have not changed for several decades.

Furthermore, we faced serious difficulty finding research sources as studies regarding basic military training for recruits and promoting and maintaining their physical fitness have rarely been performed. These tasks have been treated as one of the top secrets in Korea.

In this study, we primarily aimed to first examine the effect of 5 weeks of basic military training on various parameters (i.e., body composition, health-related physical fitness, serum lipid levels, and obesity-related hormones [these variables are required for assessing the level of physical fitness because they are included in the military test program of this study and because military applicants and active soldiers must have a generally high fitness level]) in the obese recruits as it has not been tested to determine if the current training program requires an upgrade according to the increased obese rate of military recruits. If yes, we secondarily aimed to describe the difference in effects between the current basic military training and additional training (complex capacities, e.g., including aerobic ability) on the various parameters in the obese recruits [7,8]. To obtain scientific information on the effect of the current military training as well as additional military basic training on obese recruits, we hypothesized that the scientifically examined variables reflected the 5 weeks of basic military training and additional training differently. The variables are as follows: body compositions of obese military recruits, health-related physical fitness (cardiorespiratory endurance, muscle strength, muscle endurance), serum lipid levels (total cholesterol, low-density lipoprotein [LDL], high-density lipoprotein [HDL], triglyceride), and obesity-related hormones (ghrelin and leptin). This study is intended to help military-related departments develop improved basic military training.

\section{METHODS}

\section{Subjects, basic military training, and diet regulation}

All subjects were military recruits who received a detailed explanation of the study before deciding to voluntarily participate, and informed consent was obtained from the participants. The participating recruits were randomly classified according to the obesity standard [9]. The Control ( $n=22$, Obesity group, Con) and the Exercise ( $n=26$, Obesity with exercise, Exe) groups were recruited according to this definition (Table 1).

The entire life of the recruits participating in this study was strictly controlled during the basic military training period in terms of diet intake and activities, including smoking and drinking. During the 5-week basic military training, including morning and evening, the Con was trained with the same training program (resistance and aerobic exercise training) as the normal recruit group (Table 2). Each of the Exe group participants completed the following additional exercises for four evenings a week: 5-min of stretching and squat exercise, 30-min power walking, and gymnastics without apparatus and stretching for cooling down (Table 2).

The entire diet-related consumption is severely restricted so that further caloric consumption is completely prohibited with strict control throughout the training periods. Thus, all trainees had the same food intake.

The energy expenditure of the Exe during power-walking was calculated based on the study by Ainsworth et al. and the energy expenditure estimation would be $0.0175 \mathrm{kcal} \cdot \mathrm{kg}^{-1} \mathrm{~min}^{-1} \cdot \mathrm{MET}^{-1} \times \mathrm{yMETs} \times$ weight $(\mathrm{kg})$ [10]. At the beginning, the average energy consumption was assumed to be approximately $0.0175 \mathrm{kcal} \cdot \mathrm{kg}^{-1} \mathrm{~min}^{-1} \cdot \mathrm{MET} \cdot \times 4.5 \mathrm{METs} \times 89(\mathrm{~kg}) \times 30$ $\min =210 \mathrm{kcal}$. In the case of resistance training, we assumed the results from the Harvard Medical School, reporting that a 30-min training session burns an average of 90-133 Cal [11] and the energy consumption for additional exercise gradually increases until the end of the training period. The institutional review board (AFMC-15065-IRB-15-054) approved the study.

Table 1. Participant characteristics

\begin{tabular}{lcccc}
\hline Groups $(\mathrm{n})$ & Age $(\mathrm{yr})$ & Height $(\mathrm{cm})$ & Weight $(\mathrm{kg})$ & $\mathrm{BMI}\left(\mathrm{kg} / \mathrm{m}^{2}\right)$ \\
Con $(22)$ & $19.7 \pm 0.68$ & $177.25 \pm 5.79$ & $92.99 \pm 8.56$ & $29.65 \pm 1.93$ \\
Exe (26) & $19.4 \pm 0.57$ & $173.95 \pm 4.93$ & $93.83 \pm 10.74$ & $30.25 \pm 2.41$ \\
\hline
\end{tabular}

Values are expressed as means \pm standard deviation (SD). Con, Obesity group; Exe, Obesity with exercise; BMl, body mass index; $\mathrm{n}$, number. 
Table 2. Details of the exercise programs for new recruits of the Korean Army

\begin{tabular}{|c|c|c|c|c|c|}
\hline \multirow{2}{*}{ Group } & \multicolumn{2}{|c|}{ Within the barracks } & \multicolumn{2}{|c|}{ Outside the barracks } & \multirow{2}{*}{ Additional exercise } \\
\hline & Ante Meridiem & Post Meridiem & Ante Meridiem & Post Meridiem & \\
\hline \multirow{2}{*}{ Con } & - Aerobic exercise (15 min) & Aerobic exercise & - Aerobic exercise & Returning to the Unit & \\
\hline & $\begin{array}{l}\text {-Resistant exercise } \\
\text { (using five resistant exer- }\end{array}$ & $\begin{array}{l}\text {-Resistant exercise (using } \\
\text { seven resistant exercise }\end{array}$ & $\begin{array}{l}\text { Resistant exercise (using } \\
\text { five resistant exercise }\end{array}$ & & \\
\hline Exe & Ditto & Ditto & Ditto & Ditto & $\begin{array}{l}\text { 10-min stretching } \\
\text { and squat exercise, } \\
\text { 30-min power } \\
\text { walking, and } \\
\text { gymnastics } \\
\text { (without apparatus } \\
\text { and stretching) }\end{array}$ \\
\hline
\end{tabular}

All the resistant exercise modalities are specifically described as supplementary data.

Con, Obesity group; Exe, Obesity with exercise; N/A, non-applicable.

\section{The pre- and post-test parameters}

The test parameters were divided into four main categories: isometric muscle force, physical fitness test, serum lipid levels, and hormone levels. (DS-103M, Jenix Co., Korea) (Inbody 720, Biospace Co., Korea). First, the isometric force category was divided into four lower limb isometric forces, and two upper limb isometric forces (the average values from two cross trials). For measuring the four lower limb isometric forces, two continuous measurements of the extension and flexion for $5 \mathrm{~s}$ after being fixed in the indicated seat position were done and the average values from the measurements were obtained (Curl 3530, Hur Co., Finland).

Second, the physical fitness test consisted of the following five healthrelated components: (1) the sit-up test consisted of the entire counts measured during $1 \mathrm{~min}$, (2) the 10-meter running test was the time spent for shuttling the 10-meter distance twice, (3) the endurance level in the health-related physical fitness was measured via 20-m running without lessening the speed; participants ran $20 \mathrm{~m}$ before a warning signal sounded, (4) a treadmill (Medtrack ST 55, Quinton, USA) was used for the maximal oxygen uptake test, and (5) the Rating of Perceived Exertion (RPE) was recorded every 30 seconds(;) during the test until the participant stopped. The estimated $\mathrm{VO}_{2}$ max method by Bruce et al. [12] was used to calculate RPE from the formula.

Third, the four serum lipid levels and two obesity-related hormones were calculated from the blood sample after 12 hours of fasting by the radioimmunoassay method (y-cobra 5010, Packard, USA) (Table 3).

\section{Statistical analysis}

All data are presented as the mean \pm standard deviation (S.D.). The obtained data from this study were analyzed by the methods of dependent and independent sample t-test after two-way repeated analysis of variance (ANOVA). SPSS version 20.0 was used for all statistical analyses. A value of $p<.05$ was considered to indicate a statistically significant difference in all analyses.

\section{RESULTS}

\section{Changes in body composition}

The changes of the four body compositions after the 5-week basic military training are shown in Table 3. The estimated parameters (weight, body fat, body mass index [BMI], and waist circumference) were significantly decreased after the 5-week basic military training. The Exe and Con had significantly decreased $(p<.01)$ rates for the weight, body fat, BMI, and waist circumference after the exercise (in the post-exercise test): $5 \%$ vs. $3.2 \%, 9.6 \%$ vs. $17.1 \%, 6 \%$ vs. $2.3 \%$, and $0.8 \%$ vs. $0.8 \%$, respectively. Two-way repeated ANOVA showed that all, except waist circumference, were differently changed in the interaction between group and time $(p<.001)$. All the parameters showed no significant difference between groups. 
Table 3. Changes in biochemical factors before and after undergoing the exercise program

\begin{tabular}{|c|c|c|c|c|c|c|}
\hline Parameters & & Group & Pre-test & Post-test & Source & $p$ \\
\hline \multirow[t]{12}{*}{ Body compositions } & \multirow[t]{3}{*}{ Weight (kg) } & Con & $92.99 \pm 8.56$ & $89.94 \pm 8.72^{* * * *}$ & G & .832 \\
\hline & & & & & $\mathrm{T}$ & .000 \\
\hline & & Exe & $93.25 \pm 10.31$ & $88.51 \pm 9.96^{* * *}$ & GXT & .000 \\
\hline & \multirow[t]{3}{*}{ Body fat (\%) } & Con & $28.27 \pm 6.20$ & $25.55 \pm 5.84^{* * * *}$ & G & .347 \\
\hline & & & & & $\mathrm{T}$ & .000 \\
\hline & & Exe & $31.31 \pm 6.82$ & $25.96 \pm 6.21^{* * *}$ & GXT & .000 \\
\hline & \multirow[t]{3}{*}{$\mathrm{BMI}\left(\mathrm{kg} / \mathrm{m}^{2}\right)$} & Con & $29.65 \pm 1.93$ & $28.95 \pm 1.94^{* * *}$ & G & .267 \\
\hline & & & & & $\mathrm{T}$ & .000 \\
\hline & & Exe & $30.99 \pm 2.56$ & $29.10 \pm 2.53^{* * *}$ & GXT & .000 \\
\hline & \multirow[t]{3}{*}{ W.C. (cm) } & Con & $99.46 \pm 6.21$ & $99.38 \pm 6.24^{* *}$ & G & .853 \\
\hline & & & & & $\mathrm{T}$ & .000 \\
\hline & & Exe & $99.80 \pm 6.32$ & $99.72 \pm 6.30^{* *}$ & GXT & .891 \\
\hline \multirow[t]{12}{*}{ Lower-limb isometric force } & \multirow[t]{3}{*}{$\operatorname{EXT}(\mathrm{L}, \mathrm{kg})$} & Con & $208.68 \pm 53.38$ & $247.19 \pm 53.44^{* * *}$ & G & .629 \\
\hline & & & & & $\mathrm{T}$ & .000 \\
\hline & & Exe & $215.21 \pm 56.40$ & $254.37 \pm 47.15^{* * * *}$ & GXT & .956 \\
\hline & \multirow[t]{3}{*}{$\mathrm{FLX}(\mathrm{L}, \mathrm{kg})$} & Con & $116.02 \pm 29.45$ & $137.44 \pm 33.39^{* *}$ & G & .033 \\
\hline & & & & & $\mathrm{T}$ & .000 \\
\hline & & Exe & $97.26 \pm 25.22$ & $118.86 \pm 18.94^{* * *}$ & GXT & .547 \\
\hline & \multirow[t]{3}{*}{ EXT $(\mathrm{R}, \mathrm{kg})$} & Con & $211.59 \pm 55.96$ & $261.17 \pm 66.21^{* * *}$ & G & .841 \\
\hline & & & & & $\mathrm{T}$ & .000 \\
\hline & & Exe & $212.25 \pm 55.57$ & $254.35 \pm 50.79^{* * *}$ & GXT & .349 \\
\hline & \multirow[t]{3}{*}{$\mathrm{FLX}(\mathrm{R}, \mathrm{kg})$} & Con & $122.26 \pm 28.65$ & $144.06 \pm 33.40^{* * * *}$ & G & .033 \\
\hline & & & & & $\mathrm{T}$ & .000 \\
\hline & & Exe & $104.34 \pm 26.13$ & $129.91 \pm 21.48^{* * *}$ & GXT & .547 \\
\hline \multirow[t]{6}{*}{ Upper-limb isometric force } & \multirow[t]{3}{*}{ Grip (Left, kg) } & Con & $37.32 \pm 6.45$ & $36.57 \pm 6.69$ & G & .942 \\
\hline & & & & & $\mathrm{T}$ & .457 \\
\hline & & Exe & $36.94 \pm 5.55$ & $36.73 \pm 4.68$ & GXT & .670 \\
\hline & \multirow[t]{3}{*}{ Grip (Right, kg) } & Con & $41.15 \pm 7.53$ & $39.95 \pm 8.51$ & G & .895 \\
\hline & & & & & $\mathrm{T}$ & .304 \\
\hline & & Exe & $40.34 \pm 6.83$ & $40.24 \pm 5.63$ & GXT & .382 \\
\hline \multirow[t]{15}{*}{ Health- related physical fitness } & \multirow[t]{3}{*}{ Sit up (count) } & Con & $36.86 \pm 8.81$ & $40.81 \pm 8.96^{* *}$ & G & .744 \\
\hline & & & & & $\mathrm{T}$ & .000 \\
\hline & & Exe & $38.03 \pm 6.66$ & $41.03 \pm 6.94^{*}$ & GXT & .554 \\
\hline & \multirow{3}{*}{$\begin{array}{l}20 \text { m shuttle running for } \\
\text { endurance test (count) }\end{array}$} & Con & $32.95 \pm 7.89$ & $52.59 \pm 10.01^{* * *}$ & G & .547 \\
\hline & & & & & $\mathrm{T}$ & .000 \\
\hline & & Exe & $30.65 \pm 9.47$ & $51.57 \pm 11.70^{* * *}$ & GXT & .484 \\
\hline & \multirow[t]{3}{*}{10 m running (sec) } & Con & $10.91 \pm .72$ & $10.54 \pm .87^{* *}$ & G & .831 \\
\hline & & & & & $\mathrm{T}$ & .000 \\
\hline & & Exe & $11.03 \pm .74$ & $10.50 \pm .71^{* * *}$ & $\mathrm{G} \times \mathrm{T}$ & .296 \\
\hline & \multirow[t]{3}{*}{ S.J. $(\mathrm{cm})$} & Con & $187.22 \pm 24.01$ & $189.00 \pm 27.20$ & G & .921 \\
\hline & & & & & $\mathrm{T}$ & .285 \\
\hline & & Exe & $188.03 \pm 21.13$ & $189.50 \pm 20.78$ & GxT & .918 \\
\hline & \multirow[t]{3}{*}{ Max O.U. (mL/kg/min) } & Con & $38.47 \pm 3.18$ & $42.04 \pm 2.60^{* * *}$ & G & .320 \\
\hline & & & & & $\mathrm{T}$ & .000 \\
\hline & & Exe & $36.25 \pm 3.54$ & $42.45 \pm 3.17^{* * * *}$ & GXT & .000 \\
\hline \multirow[t]{6}{*}{ Serum lipid level } & T.C. $(\mathrm{mg} / \mathrm{dL})$ & Con & $153.86 \pm 27.55$ & $140.63 \pm 21.88^{* *}$ & G & .500 \\
\hline & & & & & $\mathrm{T}$ & .000 \\
\hline & & Exe & $163.08 \pm 28.94$ & $140.76 \pm 28.71^{* *}$ & GXT & .254 \\
\hline & T.G. (mg/dL) & Con & $68.57 \pm 20.78$ & $61.57 \pm 18.68^{*}$ & G & .812 \\
\hline & & & & & $\mathrm{T}$ & .021 \\
\hline & & Exe & $68.60 \pm 15.10$ & $59.13 \pm 24.75^{*}$ & $\mathrm{G} \times \mathrm{T}$ & .720 \\
\hline
\end{tabular}


Table 3. Continued

\begin{tabular}{|c|c|c|c|c|c|c|}
\hline Parameters & & Group & Pre-test & Post-test & Source & $p$ \\
\hline & H.D.L. (mg/dL) & Con & $46.00 \pm 7.32$ & $43.68 \pm 8.93$ & G & .559 \\
\hline & & & & & $\mathrm{T}$ & .064 \\
\hline & & Exe & $44.76 \pm 5.88$ & $42.68 \pm 8.14$ & GXT & .918 \\
\hline & L.D.L. (mg/dL) & Con & $96.81 \pm 26.13$ & $85.40 \pm 23.01^{* *}$ & $\mathrm{G}$ & .680 \\
\hline & & & & & $\mathrm{T}$ & .001 \\
\hline & & Exe & $101.47 \pm 24.46$ & $86.17 \pm 26.66^{* *}$ & $\mathrm{G} \times \mathrm{T}$ & .599 \\
\hline \multirow[t]{6}{*}{ Obese related hormone } & Ghrelin (pg/mL) & Con & $989.40 \pm 271.34$ & $1,137.31 \pm 248.69^{* * * *}$ & G & .163 \\
\hline & & & & & $\mathrm{T}$ & .000 \\
\hline & & Exe & $1,091.32 \pm 252.34$ & $1,225.20 \pm 271.50^{* *}$ & GxT & .849 \\
\hline & Leptin $(\mathrm{pg} / \mathrm{mL})$ & Con & $8.73 \pm 4.46$ & $5.86 \pm 1.95^{* * *}$ & G & .183 \\
\hline & & & & & $\mathrm{T}$ & .000 \\
\hline & & Exe & $8.31 \pm 4.35$ & $4.08 \pm 2.02^{* * * *}$ & $\mathrm{G} \times \mathrm{T}$ & .234 \\
\hline
\end{tabular}

Values are means \pm standard deviation.

Con, Obesity group; Exe, Obesity with exercise; BMI, body mass index; W.C., waist circumference; EXT (L), extension light; FLX (R), flexion right; EXT (L), extension light; FLX (L), flexion light; Grip, grip strength; S.J., standing jump; Max O.U., maximal oxygen uptake; T.C., total cholesterol; T.G., Triglyceride; HDL, high density lipoprotein; LDL, low density lipoprotein; $G$, group; $T$, time; $G \times T$, group $\times$ time.

${ }^{*} p<.05,{ }^{* *} p<.01$, and ${ }^{* * *} p<.001$ indicating statistical significances between the group pre- and post-, ${ }^{*} 20 \mathrm{~m}$ shuttle running for endurance test, increasing the number of times for the pointed $20 \mathrm{~m}$.

\section{Upper and lower limb isometric force and health- related physical fitness}

There was no significant difference in grip strength between the Con and Exe (Table 3), and no significant difference was shown in the actual power promotion of the ambi-grip strength by the effect of the military exercise program. Group difference between the left and right grip strength was not statistically significant. Similarly, time difference and interaction between group and time were not significant.

Entire lower limb isometric force-related parameters significantly were improved by the training program $(p<.01)$. Time also affected the lower limb power improvement $(p<.001)$. Left and right flexion force showed significant differences between the Con and Exe $(p<.05$, Group difference). Interaction between time and group change showed no significant difference in the isometric force-related parameters.

Out of the five health-related physical fitness components, the standing jump showed no significant difference for either group, even though all parameters were significantly different $(p<.05)$, especially the aerobic capacity-related parameters, such as the 20-m shuttle running for the endurance test and the maximal oxygen uptake test, which were remarkably promoted by the training program $(p<.01)$. Except the standing jump, all the health-related physical fitness components showed significant difference between pre- and post-test values $(p<.001)$. The significant difference of the interaction between time and group was shown only in the maximal oxygen uptake $(p<.001)$.

\section{Laboratory test results}

Four serum lipid-related parameters (total cholesterol, triglyceride, HDL, and LDL) and obesity-related hormones (ghrelin and leptin) were examined (Table 3). For the serum lipid levels, all parameters, except HDL, were significantly improved (8.5-20\%) by the 5-week training $(p<.05)$. There was no statistical difference between the Con and Exe as well as in the interaction between group and time.

For obesity-related hormones between the pre- and post-5-week basic military exercise training, only ghrelin was not significantly different between the pre- and post-training $(p<.01)$. The interaction between the group and time also showed no significant difference.

\section{DISCUSSION}

We found that the 5-week military training for new 48 military recruits (22 Con vs. 26 Exe) affects the maintenance and improvement in body compositions, lower limb isometric force, health-related physical fitness, serum lipid levels, and obesity-related hormones (especially for the Exe). There were significant differences in four body compositions (weight, body fat, BMI, and waist circumference) shown between the pre- and post-tests for both the Con and Exe $(p<.01)$. Muscle endurance, cardiovascular capacity, and lower limb isometric force as the health-related physical fitness parameters showed significant differences between the pre- and post-tests in both the Con and Exe $(p<.05)$. Only the total cholesterol, triglyceride, and LDL levels were decreased $(p<.05)$ between 
the pre- and post-tests for both groups. Significant increases in ghrelin were observed between the pre- and post-tests for the Con and Exe $(p<.01)$. Conversely, significant decreases were found in the leptin levels for the same condition $(p<.001)$. Significant changes were only noted in lower ambi-limb isometric force flexion values from training between the Con and Exe $(p<.05)$.

\section{Body compositional changes and the importance of these results}

Many studies reported that a 5-10\% decrease in body weight induces significant improvement in heart disease-related risks caused by factors such as decreased blood pressure, total cholesterol, triglyceride, and fasting glucose levels $[13,14]$. Exercise intervention is an indispensable way for optimizing cardiovascular system-related metabolism and preventing chronic disease-causing risk factors [15].

Considering the purpose of the exercise, the target-adjusted exercise program development is indispensable, and those data are usually collected or planned based on the previous studies. This study on the effects of basic training of military recruits is the first of its kind. In Korea, case studies regarding the data sourced from the military force are rare because of the extremely tense situation of the Korean peninsula (almost 70 years of ceasefire have passed, but a full-fledged war can occur any time). As a result, many studies have been confidential, and military recruit-related data have usually been excluded. This is, therefore, the first report disclosing the contents of the basic military training for militaryconscripted applicants. The physical fitness training program used in this study has been also inter-effected with few other military programs performed in Korea for improving the contents of programs [16]. The subjects (obese military recruits) of this study were trained under highly intensive conditions (which involved controlled diet pattern, exercise, and regular lifestyle during the training period), which had positive effects in the Con and Exe.

To construct a more powerful military force, the ministry of National Defense tries to reform with resolute will and this is a basic step. Because of the affluent social situation, recruits that are more obese began entering military bases, which seriously contributed to the decrease in the quality of the military force. As the military candidates who are recruited in recent years also have a lower physical fitness level than previous candidates, the existing exercise training does not involve an adequate training program to make up for the insufficient physical fitness for the current candidates even though the current standard 5-week basic mili- tary training is an already intensive physical training program. The effectively higher parameter levels in the Exe than in the Con in this study evidently demonstrate the necessity of an improved training program.

In our results regarding body composition, all the parameter results in the Con and Exe are consistent with the previous studies, and the additional exercise program applied to the Exe had significantly better results, which should be seriously considered to include the novel basic military exercise program for the next generation of recruits.

\section{Physical fitness-related results from this study}

The present results show that the aerobic capacity-related parameters (e.g., the 20-m shuttle running endurance test and maximal oxygen uptake) were affected by the 5-week basic military exercise program for recruits, rather than resistant force-related parameters, such as the grip isometric force test (Table 3). We suggest emphasizing the important results of this study to develop a program that includes the pull-up for improving grip strength (since there were no significant differences shown between the Con vs. Exe). A full-scale war may possibly occur if the second Korean War occurs, which will surely involve hand-to-hand fight combat. We suggest improving the overall parameters of the basic military program for recruits before a war occurs.

In the normal Con recruits or even Exe groups, there was no significant change shown in the standing jump that indicates physical agility capacity, which is possibly dominated by a neuromuscular-related viviparous capacity. We suggest that the innate capacity for agility should be considered for candidates, especially when recruiting for the specified task force team within the military force or agility-demanding fields of the military force.

\section{Changes in serum lipid and obesity-related hormone levels}

In our data, three serum lipid levels (total cholesterol, triglyceride, and LDL levels) were significantly decreased, except for HDL $(p<.05)$, which is consistent with a previous report [14], even though the exercise intervention effects seem to be dependent on the different modalities, intensities, and frequencies of the exercise programs.

With regard to obesity-related hormones, the Con and Exe showed significantly increased ghrelin levels, and the leptin levels were inversely and proportionally decreased within both groups $(p<.01)$. This is in line with the general notion about the opposite role of these hormones (i.e., ghrelin stimulates appetite and leptin decreases during exercise; however, 
excessive exercise reversely affects appetite). Conversely, these hormone levels also have been inconsistent in previous results that were dependent on different exercise types, intensities, durations, frequencies, and participant types. Regarding the lack of significant differences between the pre- and post-exercise results of these two hormones, we suggest that this is caused by a big difference in the study participants.

We, thus, suggest that a target-adjusted exercise intervention should be used according to optimal frequencies, modalities, and intensities. If more large-scale populations are available, the non-significant difference shown in the results (possibly caused from the large test result differences between individuals) for the values differing between pre- and postexercise intervention will possibly produce results different from the current ones.

\section{CONCLUSIONS}

Current programs for improving the military recruits' health have not shown practical effects on promoting health and physical fitness that could contribute to combat power. To terminate the war once it occurs, large-scale armed conflict by combat military force is inevitable, even though both sides are fully armed with the avant-garde weapons of the modern war. Physically competitive soldiers should, thus, be indispensably trained and/or maintained. It is essential to develop policies to support systematic science-based studies using beneficial information since they will possibly contribute to raising the competitive physical fitness of each soldier, which will significantly assist national security.

\section{CONFLICT OF INTEREST}

No potential conflict of interest relevant to this article was reported.

\section{AUTHOR CONTRIBUTION}

Conceptualization: JH Yoon; Data curation: SH Park; Methodology: JH Yoon, BW Kim; Project administration: BW Kim; Writing- original draft: SG Seo; Writing review and editing: H Jee.

\section{ORCID}

Hyunseok Jee https://orcid.org/0000-0003-4702-0570

\section{REFERENCES}

1. Alegria Ezquerra E, Castellano Vazquez JM, Alegria Barrero A. Obesity, metabolic syndrome and diabetes: cardiovascular implications and therapy. Rev Esp Cardiol. 2008;61(7):752-64.

2. Akil L, Ahmad HA. Relationships between obesity and cardiovascular diseases in four southern states and Colorado. J Health Care Poor Underserved. 2011;22(4):61-72.

3. Pihlainen K, Vaara J, Ojanen T, Santtila M, Vasankari T, et al. Effects of baseline fitness and BMI levels on changes in physical fitness during military service. J Sci Med Sport. 2020;S1440-2440(19)31497-5.

4. Sanderson PW, Clemes SA, Biddle SJ. The correlates and treatment of obesity in military populations: a systematic review. Obes Facts. 2011; $4(3): 229-37$.

5. Seo SG, Ji YS, Woo DY, Yoon JH. Effects of 6 weeks plyometric training on isokinetic leg strength, agility, and vertical jump and in Korea ROTC cadets. J Korean Soc Living Environ Sys. 2016;23(4):519-26.

6. Kim K, Kim J, Ko J. The analysis of energy consumption from physical activity on weekly basic military training for KAFA cadet candidates. KJMAS. 2014;70(3):165-80.

7. Knapik JJ, Reynolds KL, Harman E. Soldier load carriage: historical, physiological, biomechanical, and medical aspects. Mil Med 2004; 169(1):45-56.

8. Knapik JJ, Rieger W, Palkoska F, Camp SV, Darakjy S. United states army physical readiness training: rationale and evaluation of the physical training doctrine. J Strength Cond Res. 2009;23(4):1353-62.

9. WHO/IASO/IOTF. The Asia-Pacific perspective: redefining obesity and its treatment. Health Communications, Australia: Melbourne. 2000.

10. Ainsworth B, Haskell W, Leon A, Jacobs D, Montoye H, et al. Compendium of physical activities: classification of energy costs of human physical activities. Med Sci Sports Exerc. 1993;25(1):71-80.

11. https://www.health.harvard.edu/newsweek/Calories-burned-in30-minutes-of-leisure-and-routine-activities.htm

12. Bruce RA, Kusimi F, Hosmer D. Maximal oxygen intake and nomographic assessment of functional aerobic impairment in cardiovascular disease. Am Heart J. 1973;85(5):546-62.

13. Lau DC, Douketis JD, Morrison KM, Hramiak IM, Sharma AM. 2006 Canadian clinical practice guidelines on the management and prevention of obesity in adults and children [summary]. CMAJ. 2007; 176(8):S1-S13. 
14. Weiss EP, Albert SG, Reeds DN, Kress KS, Mcdaniel JL, et al. Effects of matched weight loss from calorie restriction, exercise, or both on cardiovascular disease risk factors: a randomized intervention trial. Am J Clin Nutr. 2016;104(3):576-86.

15. Pedersen BK, Saltin B. Exercise as medicine-evidence for prescribing exercise as therapy in 26 different chronic diseases. Scand J Med Sci Sports. 2015;25(3):1-72.

16. Kim K, Kim J, Lee Y, Park J, Ko J. The analysis of energy consumption from physical activity on weekly basic military training for KAFA cadet candidate. Korean J Mil Art Sci. 2014;70(3):165-80. 